\title{
Development of frequency-dependent ocean wave directional distributions
}

\author{
Abushet Simanesew ${ }^{\mathrm{a}}$, Harald Krogstad ${ }^{\mathrm{b}}$, Karsten Trulsen ${ }^{\mathrm{a}, *}$, José Carlos Nieto \\ Borge $^{c}$ \\ ${ }^{a}$ Department of Mathematics, University of Oslo, PO Box 1053 Blindern, NO-0316 Oslo, \\ Norway \\ ${ }^{b}$ Department of Mathematical Sciences, NTNU, NO-7491 Trondheim, Norway \\ ${ }^{c}$ Signal Theory and Communications Department, Superior Politechnic School, University \\ of Alcalá, Alcalá de Henares, 28805, Madrid, Spain
}

\begin{abstract}
The paper discusses the development of a frequency dependent directional spread from an initial condition of frequency-independence. The study applies basin directional measurements from the Maritime Research Institute Netherlands (MARIN), simulated data from a nonlinear wave equation and field measurements from the Ekofisk field. The basin experiments and numerical simulations are initialized with a JONSWAP spectrum with frequency-independent directional distributions. In both cases we observe the development of a strong frequency-dependence of the directional spread. The numerical simulations suggest that static nonlinear contributions to the surface elevation partially explain the behavior below the spectral peak in accordance with [1]. There are also dynamic nonlinear contributions on both sides of the spectral peak.
\end{abstract}

Keywords: Frequency dependent directional distribution

\section{Introduction}

In Gaussian linear wave theory (LWT), the ocean wave field is characterized by the directional spectrum

$$
E(\omega, \theta)=S(\omega) D(\theta, \omega),
$$

\footnotetext{
* Corresponding author

Email address: karstent@math.uio.no (Karsten Trulsen)
}

Preprint submitted to Journal of Applied Ocean Research 
where $S$ is the frequency spectrum, $D$ the directional distribution, $\omega$ the angular frequency and $\theta$ is the direction [2]. If the required assumptions are made, the directional spectrum can easily be extracted from ocean wave data recorded 5 using instruments ranging from the simplest single point triplet and gauge arrays to the more recent stereo video-based [3, 4, 5, 6] and remote-sensing [7] systems. Many studies dealing with the frequency dependent behavior of ocean wave directional distribution often assume the $\cos -2 s$ distribution [8] as a primary choice, and express the spreading parameter, $s$, in terms of the dimensionless frequency to optimize the fit to measured directional characteristics. Proposed parameteric representations can be found from the recent studies of [9, 10, 11] and early work of [12, 13, 14] for practical applications. However, there are also several other idealized directional distributions with equivalent parameters as in the $\cos -2 s$ distribution, suitable to describe the frequency dependence of the directional spread, or the circular standard deviation of $D,[15]$. A more comprehensive review of many of the parameterizations are given in [16]. Almost all analysis based on ocean wave data show that the directional spread is strongly dependent on frequency, with minimum spread near the spectral peak.

Since the pioneering work of Hasselmann [13], the frequency dependence of the angular distribution has been speculated to be due to the dominance of nonlinear interactions over generation effects. Second order spectral contributions derived from perturbation expansion of the surface elevation up to the $4^{\text {th }}$ order in wave steepness [e.g. 17], reveal nonlinear influence on the shape of the directional spread. In particular, the difference-frequency contribution is the principal cause for the strong increase in the spread, and the discrepancy between the RMS and the linear wave theory wavenumber below the spectral peak [1]. The three-dimensional MNLS simulations of [18], carried out to study the establishment of high frequency power-law and the corresponding shift in the spectral peak, lead to the same conclusion.

We are not aware that basin wave spectra have yet been investigated whether they develop a frequency dependent directional spread on their own. However, in advanced basin wave makers, calibration of directional seas sometimes employs 
realistic frequency dependent directional distributions [e.g. 19].

In the present paper, we take advantage of available experimental data to investigate the development of frequency dependent directional spread. The corresponding numerical simulations are done using the modified nonlinear Schrödinger equation [20, 21, 22, 23, 24].

We also present observations from the Ekofisk laser array, discuss data analysis methods, and observe bimodal nature of the directional distributions [25, 9, 26, 27].

The next section presents the theoretical background and analysis methods. Sec. 3 and 4 summarize data sources and results, respectively, followed by the conclusions in Sec. 5 .

\section{Background theory}

\subsection{Wave spectra}

We consider a zero mean, weakly stationary and homogeneous ocean surface, $z=\eta(\mathbf{x}, t)$, where $\mathbf{x}=(x, y)$ is the horizontal position, $t$ is time, and the $z$ axis pointing upwards. The spectral representation of $\eta$ is a stochastic Fourier integral over the wavenumber-frequency $(\mathbf{k}, \omega)$-space,

$$
\eta(\mathbf{x}, t)=\int_{\mathbf{k}, \omega} e^{i(\mathbf{k} \cdot \mathbf{x}-\omega t)} d Z(\mathbf{k}, \omega) .
$$

The Fourier transform in this context, $Z$, is called the spectral amplitude. From the properties of $Z$, the covariance function, $\rho(\mathbf{x}, t)$, and the spectrum of the surface are related by the integral

$$
\rho(\mathbf{x}, t)=\mathbb{E}[\eta(\mathbf{x}, t) \eta(\mathbf{0}, 0)]=\int_{\mathbf{k}, \omega} e^{i(\mathbf{k} \cdot \mathbf{x}-\omega t)} d \chi(\mathbf{k}, \omega) .
$$

It is convenient to write $d \chi(\mathbf{k}, \omega)=S(\omega) \phi_{\omega}(\mathbf{k}) d \omega d \mathbf{k}$. Here $\phi_{\omega}(\mathbf{k})$ is the wavenumber distribution at frequency $\omega$, normalized so that $\int_{\mathbf{k}} \phi_{\omega}(\mathbf{k}) d \mathbf{k}=1$ for all $\omega \in \mathbb{R}$, where $d \mathbf{k} \equiv d k_{x} d k_{y}$ is a short-hand notation. In the simplest case with only linear free waves, $\mathbf{k}$ and $\omega$ are connected by the dispersion relation,

e.g. $\omega=\sqrt{g|\mathbf{k}| \tanh (|\mathbf{k}| h)}, k_{x}=|\mathbf{k}| \cos \theta, k_{y}=|\mathbf{k}| \sin \theta$, and $\phi_{\omega}$ may be 
written as $\phi_{\omega}(\mathbf{k})=\delta\left(|\mathbf{k}|-k_{L W T}(\omega)\right) D(\theta, \omega)$, where $k_{L W T}$ is the solution of the dispersion relation for a fixed $\omega$, and $D(\theta, \omega)$ is the angular distribution located at the intersection of the dispersion manifold and the plane $\omega=$ const.

Following the discussion in [2], $\phi_{\omega}(\mathbf{k})$ will in general have contributions also off the dispersion manifold. Being a two-dimensional distribution, it is reasonable to consider its basic moments up to second order,

$$
\left\langle\left(k_{x}\right)^{i}\left(k_{y}\right)^{j}\right\rangle(\omega)=\int_{\mathbf{k}}\left(k_{x}\right)^{i}\left(k_{y}\right)^{j} \phi_{\omega}(\mathbf{k}) d \mathbf{k}, \quad 0 \leq i+j \leq 2 .
$$

Apart from rather crude large-area remote-sensing systems, direct field measurements of $\phi_{\omega}(\mathbf{k})$ are out of reach. However, estimates of these moments are possible from single point surface heave/pitch/roll (HPR) systems. The method is well established, but unfortunately, the leading five moments are far from determining the actual shape of $\phi_{\omega}$, as illustrated by the following two possible solutions. The first solution is a two-dimensional Gaussian distribution, whereas the second is a quasi-LWT solution where the wave number is estimated from the data without enforcing by the dispersion relation [2, 28]. The first has hardly been used, but the second has become a standard for heave/pitch/roll systems where four Fourier coefficients of $D(\theta, \omega)$ may be uniquely obtained from the moments.

The preferred object in research, in particular in remote sensing is the wavenumber spectrum

$$
\Psi(\mathbf{k}) d \mathbf{k}=2 \int_{d \mathbf{k}, \omega>0} d \chi(\mathbf{k}, \omega)=2 \int_{\omega>0} S(\omega) \phi_{\omega}(\mathbf{k}) d \mathbf{k} d \omega .
$$

However, in the general case, $\Psi(\mathbf{k})$ will contain contributions from several $\phi_{\omega^{-}}$ distributions for each $\mathbf{k}$, and there is no way to discriminate between contributions from linear and nonlinear waves without further information about the $\phi_{\omega}$-functions. Inversion from $\Psi$ back to $\chi$ is thus only possible assuming the dispersion relation, such as $\sigma(|\mathbf{k}|)=\sqrt{g|\mathbf{k}| \tanh (|\mathbf{k}| h)}$,

$$
d \chi(\mathbf{k}, \omega)=\frac{1}{2}[\Psi(\mathbf{k}) \delta(\omega-\sigma(|\mathbf{k}|))+\Psi(-\mathbf{k}) \delta(\omega+\sigma(|\mathbf{k}|))] d \mathbf{k} d \omega
$$

The transformation from the wavenumber spectrum to the commonly used 
directional spectrum, $E(\omega, \theta)=S(\omega) D(\theta, \omega)$, is carried out by change in variables:

$$
E(\omega, \theta)=S(\omega) D(\theta, \omega)=\Psi(|\mathbf{k}|(\omega), \theta)|\mathbf{k}|(\omega) \frac{d|\mathbf{k}|(\omega)}{d \omega} .
$$

It is convenient to normalize $S$ and $\Psi$ so that

$$
\operatorname{Var}[\eta]=\int_{\mathbf{k}, \omega} d \chi(\mathbf{k}, \omega)=\int_{\mathbf{k}} \Psi(\mathbf{k}) d \mathbf{k}=2 \int_{0}^{\infty} S(\omega) d \omega .
$$

The angular distribution function $D(\theta, \omega)$ may be viewed as a probability distribution function distributed over the direction $\theta$ of $\mathbf{k}$, often stated in terms of its Fourier series expansion,

$$
D(\theta, \omega)=\frac{1}{2 \pi}\left[1+2 \sum_{n=1}^{\infty}\left[a_{n}(\omega) \cos n \theta+b_{n}(\omega) \sin n \theta\right]\right] .
$$

The main parameters of the distribution are expressed in terms of the Fourier coefficients $a_{1}(\omega)$ and $b_{1}(\omega)$ [see 29, 2]. The mean direction is given by $\theta_{1}(\omega)=$ $\operatorname{atan} 2\left(b_{1}(\omega), a_{1}(\omega)\right)$, whereas the directional spread, $\sigma_{1}(\omega)=\sqrt{2\left(1-r_{1}(\omega)\right)}$, where $r_{1}(\omega)=\sqrt{a_{1}(\omega)^{2}+b_{1}(\omega)^{2}}$. The cos-2s distribution is a favored choice in modeling directional wave fields:

$$
D(\theta)=D_{s}(s) \cos ^{2 s} \frac{\left(\theta-\theta_{1}\right)}{2}, \quad D_{s}(s)=\frac{1}{\pi} 2^{2 s-1} \frac{\Gamma^{2}(s+1)}{\Gamma(2 s+1)}, \quad \sigma_{1}=\sqrt{\frac{2}{s+1}},
$$

here $s$ can be constant or in accordance with one of the empirical frequency dependent forms proposed by e.g. [14, 13] or [9]. For example, the Mitsuyasu parameterization [14] takes the form

$$
s(\omega)=\left\{\begin{array}{lll}
s_{p}\left(\frac{\omega}{\omega_{p}}\right)^{5} & \text { if } & \omega \leq \omega_{p}, \\
s_{p}\left(\frac{\omega}{\omega_{p}}\right)^{-2.5} & \text { if } & \omega>\omega_{p},
\end{array}\right.
$$

${ }_{65}$ where $s_{p}=11.5\left(U / c_{p}\right)^{-2.5}, U$ is the wind speed and $c_{p}$ the phase speed at the spectral peak.

It is possible to relate the Fourier coefficients directly to the moments of $\phi_{\omega}$-functions, 2]. In particular, introducing the mean wavenumber, $\langle\mathbf{k}\rangle(\omega)=$ 
$\left\langle\left(k_{x}, k_{y}\right)\right\rangle$, and RMS wavenumber, $k_{R M S}(\omega)=\left\langle k_{x}^{2}+k_{y}^{2}\right\rangle^{1 / 2}$, we obtain the general expressions

$$
\theta_{1}(\omega)=\angle \mathbf{r}(\omega), \quad \sigma_{1}(\omega)=\sqrt{2(1-|\mathbf{r}|(\omega))}, \quad \mathbf{r}(\omega)=\frac{\langle\mathbf{k}\rangle(\omega)}{k_{R M S}(\omega)} .
$$

These definitions can be used independently of any additional assumptions beyond stationarity and homogeneity. We also employ the useful Check Ratio, $C R(\omega)=\frac{k_{R M S}(\omega)}{k_{L W T}(\omega)}$, where $k_{L W T}(\omega)$ is obtained from the dispersion relation.

\subsection{Methods of data analysis}

Stochastic wave data analysis mostly applies Fourier-based methods. This is ideal for Gaussian linear wave theory, where any linear and shift invariant filter, $\eta(\mathbf{x}, t) \rightarrow \xi(\mathbf{x}, t)$, may be expressed as

$$
\xi_{m}=\int_{\mathbf{k}, \omega} T_{m}(\mathbf{k}, \omega) e^{i\left(\mathbf{k} \cdot \mathbf{x}_{m}-\omega t\right)} d Z(\mathbf{k}, \omega) .
$$

Here $T_{m}$ is the transfer function and $e^{i \mathbf{k} \cdot \mathbf{x}_{m}}$ the location exponential. A first step in the analysis, following the data inspection and restoration, is to estimate the auto- and cross-spectral matrix, $\Sigma(\omega)=\left[\sigma_{m n}\right]_{m, n=1}^{M}$, defined by

$$
\sigma_{m n}(\omega)=\int_{\mathbf{k}} T_{m}(\mathbf{k}, \omega) T_{n}^{H}(\mathbf{k}, \omega) e^{i \mathbf{k} \cdot\left(\mathbf{x}_{m}-\mathbf{x}_{n}\right)} d \chi(\mathbf{k}, \omega), m, n=1, \ldots, M,
$$

where $H$ stands for Hermitian transposed [2]. The transfer functions in the integral Eq. 14 including the location exponentials may be expressed in terms of $\omega$ and $\theta$ as $T_{i}(\omega, \theta)=R_{i}(\omega) h_{i}(\omega, \theta)$, where $R_{i}(\omega)$ and $h_{i}(\omega, \theta)$ contain the direction-independent and dependent parts, respectively [30]. If we further assume a directional spectrum of the form given in Eq. 7 then Eq. 14 may be rewritten as

$$
\boldsymbol{\Sigma}(\omega)=S(\omega) \mathbf{R}(\omega) \boldsymbol{\Phi}(\omega) \mathbf{R}^{H}(\omega), \quad \mathbf{R}=\operatorname{diag}\left(R_{1}, \ldots, R_{N}\right)
$$

where

$$
\boldsymbol{\Phi}(\omega)=\int_{0}^{2 \pi} \mathbf{h}(\omega, \theta) \mathbf{h}^{H}(\omega, \theta) D(\theta, \omega) d \theta
$$

We refer to 2] for computational details, but recall that estimation of $\boldsymbol{\Sigma}$ is made by averaging the products of the discrete Fourier transform of the time 
series provided the data are well-sampled. This means that the data records are considerably longer than the temporal correlation, and the sampling frequency is well above the most energetic parts of the spectrum. In addition, data windowing and spectral averaging are used for decreasing spectral leakage and enhance the quality of the estimate.

There are many different estimation techniques for wave spectra, each having their own principle of analysis and merits [31]. We consider the estimation of directional wave spectra from spatial arrays of wave gauges applying the maximum likelihood (ML) method, the iterative maximum likelihood (IML) method, the heave/pitch/roll (HPR) method, and directly from $\chi(\mathbf{k}, \omega)$ when feasible. The ML estimate has the form [32]

$$
D_{M L}(\theta, \omega)=\frac{\kappa}{\mathbf{h}^{H}(\omega, \theta) \Phi^{-1}(\omega) \mathbf{h}(\omega, \theta)},
$$

where

$$
\kappa^{-1}=\int_{0}^{2 \pi}\left(\mathbf{h}^{H}(\theta, \omega) \boldsymbol{\Phi}^{-1} \mathbf{h}(\theta, \omega)\right)^{-1} d \theta .
$$

Data redundancy may sometimes require use of generalized inverses. However, cross-spectra computed from a certain $D(\theta)$ and then used to compute $D_{M L}$ from Eq. 17 do not always match. Therefore, Pawka [33] introduced an iterative improvement of ML method called iterative maximum likelihood (IML) method, later modified to an iterative scheme of the form

$$
D_{n+1}=D_{n}+\omega_{R}\left[\hat{D}_{M L}-M\left(D_{n}\right)\right] \quad \text { with } \quad D_{0}=\hat{D}_{M L} .
$$

In Eq. 19. $\hat{D}_{M L}$ is the ML estimate based on the data, $M\left(D_{n}\right)$ the ML estimate based on cross-spectrum of $\hat{D}_{M L}, \omega_{R}$ a relaxation parameter, and so $D_{n+1}$ is normalized to have integral equal 1, 32, 34].

One may alternatively analyze three sensors as HPR data by adding a plane through the three measurements and approximate the surface elevation, $\eta$, and slopes, $\frac{\partial \eta}{\partial x}$ and $\frac{\partial \eta}{\partial y}$ at the center of gravity. In this case, we get single-point triplet measurements which may be analyzed using the standard buoy processing techniques [see, 35, p.184], leading to, among others, the Burg [36] maximum 
entropy (ME-B) and the Shannon [37] maximum entropy (ME-S) directional distributions.

\section{The different data sources}

\subsection{Laboratory measurements}

90

Data for this study were collected by MARIN as part of the On board Wave and Motion Estimator (OWME) project. The experiment was conducted between Dec. 10 to Dec. 13, 2007, and was initiated with the main objective of developing an on board decision support system for vessel motions prediction. A detailed description of the facilities and experiments is presented in [38].

Model experiments at a scale of $1: 70$ were performed in the $40 \mathrm{~m}$ long and $170 \mathrm{~m}$ wide MARIN seakeeping and manoeuvring basin. The wave measurements were carried out using a $1.8 \mathrm{~m}$ by $1.8 \mathrm{~m} 10 \times 10$ wave probe array of grid size $0.2 \mathrm{~m}$ in both directions as shown in Fig. 1a. The array was connected to a movable carriage which could be moved to various locations in the basin between tests, each such location will in the following be referred to as array location. Arrangement of the wave gauge arrays, as well as their layouts throughout the $5 \mathrm{~m}$ deep (350m in full scale) model basin is shown in Fig. 1b the main wave propagation direction is along the positive $x$-axis. The waves are generated by a flap type wave maker at one end and damped by an absorbing beaches on the opposing end. The wave maker is located $-740 \mathrm{~m}$ from the $y$-axis.

The wave maker was programmed with a JONSWAP spectrum with a directional distribution independent of frequency. The JONSWAP spectrum was generated with peakedness factor $\gamma=3.3$, significant wave height $H_{s}=2.5 \mathrm{~m}$, a peak period of $T_{p}=9 \mathrm{~s}$. Three different directional distributions were employed, narrow $\left(\sigma_{1 I}=5.7^{\circ}\right)$, medium $\left(\sigma_{1 I}=12.5^{\circ}\right)$ and broad $\left(\sigma_{1 I}=19.1^{\circ}\right)$. The directional distribution was similar to the standard cos- $2 s$-distribution introduced, see [8]. The spectral peak frequency in the measurement was $f_{p}=0.113 \mathrm{~Hz}$, corresponding to $k_{p}=0.0514 \mathrm{rad} / \mathrm{m}$, wavelength $\lambda_{p}=122 \mathrm{~m}$ and dimensionless 


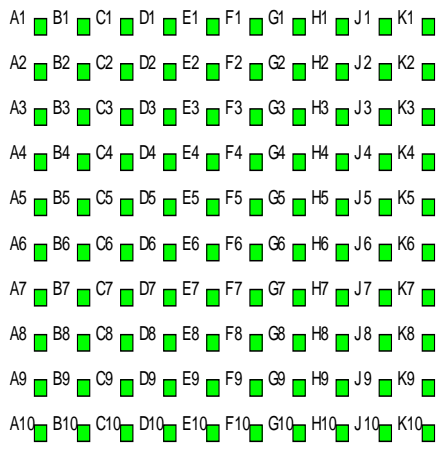

(a) Resistance type wave probes arranged in a square with sides 126 metres (full scale).

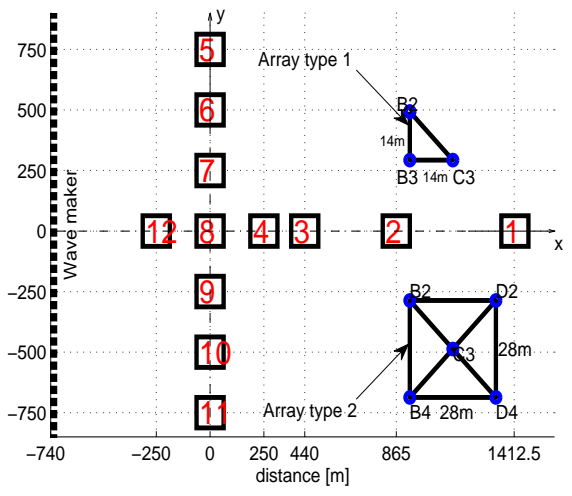

(b) Full scale wave gauge array locations. The distance between the wave maker and the center of location 12 is $490 \mathrm{~m}$.

Figure 1: Wave gauge array locations and probe arrangement

depth $k_{p} h=19$. In the following, we define the steepness $\epsilon$ as $\epsilon=k_{p} \sqrt{2\left\langle\eta^{2}\right\rangle}$, accordingly the steepness in MARIN data is found to be $\epsilon=0.05$.

Analysis results presented in Sec. 4.1 are based on measurements either from array type 1 or from array type 2. The former formed by probes B2, B3 and C3, while the latter formed by probes B2, B4, C3, D2 and D4 as depicted in Fig. 1b. The target location in the basin will be indicated in the caption when needed. The noise level in the auto-spectra is about $40 \mathrm{~dB}$ below the peak and limits the useful part of the spectra to an interval, $\left[0,4 \omega_{p}\right]$.

Due to some noise of unknown origin, neighboring data series show lack of coherence in the cross-spectra above twice the spectral peak. The noise in the individual time series implies a fast drop in coherence in the cross-spectrum and a corresponding poor quality of the estimated wave parameters. In addition, data from managable subarrays from the full arrays suffer from spatial aliasing and limits the usable frequency range for the directional analysis even further. In the MARIN probe set-up, the smallest leg in the array is $14 \mathrm{~m}$, resulting in a limiting wavelength of $28 \mathrm{~m}$. The corresponding frequency is $0.24 \mathrm{~Hz}$, or about 
two times the spectral peak frequency.

\subsection{Field measurements}

The Ekofisk laser array consists of four down-looking Optech ${ }^{\mathrm{TM}}$ lasers mounted on a bridge connecting the Kilo and Bravo platforms near the Ekofisk complex. The lasers are placed at the four corners of a $2.6 \mathrm{~m}$ by $2.6 \mathrm{~m}$ square located approximately 20 meters above the mean surface of a $70 \mathrm{~m}$ deep sea.

The system has been designed by the Norwegian Meteorological Institute in cooperation with the University of Miami, and the data collection is carried out under the operational responsibility of ConocoPhillips Inc. The lasers use continuous $5 \mathrm{~Hz}$ sampling frequency which was down-sampled to $1.7 \mathrm{~Hz}$ for time series of duration 20 minutes 39]. Sophisticated data check and restoration techniques have been implemented on the raw data [40]. The present analysis is limited to records with moderate to high sea states, that is, $H_{s} \geq 3.5 \mathrm{~m}$. The spectral peak frequency is found to be $f_{p}=0.12 \mathrm{~Hz}$, the dispersion relation gives $k_{p}=0.0616 \mathrm{rad} / \mathrm{m}, \lambda_{p}=2 \pi / k_{p}=102 \mathrm{~m}$ and thus $k_{p} h=4.3$.

145 Even if the full array consists of four lasers, the results are virtually unchanged when using only three lasers because of the array's compact size. The shortest leg in the array is $2.6 \mathrm{~m}$, resulting in a limiting wavelength $\lambda_{S A}=5.2 \mathrm{~m}$. The corresponding frequency, $f_{S A} \approx 0.56 \mathrm{~Hz}=4.4 f_{p}$, which is well below the temporal Nyquist limit $f_{s}=0.85 \mathrm{~Hz}$.

\subsection{Numerical model}

We shall employ a modified nonlinear Schrödinger model that describes the evolution of the surface elevation using the evolution of its complex envelope, $B$. In this section all expressions are non-dimensionalized by a characteristic wavenumber $\left(k_{c}\right)$ and the corresponding characteristic angular frequency $\left(\omega_{c}\right)$. In practice we let the characteristic frequency be the peak frequency of the initial spectrum. Assuming a two dimensional, irrotational flow of an incompressible inviscid fluid, we expand the velocity potential $\phi$ and surface elevation $\eta$ of the 
free surface in harmonic series

$$
\phi=\bar{\phi}+\frac{1}{2}\left(A e^{i(x-t)}+A_{2} e^{2 i(x-t)}+A_{3} e^{3 i(x-t)}+\cdots+\text { c.c. }\right),
$$

and

$$
\eta=\bar{\eta}+\frac{1}{2}\left(B e^{i(x-t)}+B_{2} e^{2 i(x-t)}+B_{3} e^{3 i(x-t)}+\cdots+\text { c.c. }\right) .
$$

Here c.c. denotes the complex conjugate, $(x, y)$ and $z$ are horizontal and vertical coordinates, $t$ is time and $\bar{\eta}, B_{2}$ and $B_{3}$ are the zeroth, second and third harmonic bound waves. They are slowly varying functions of space and time given by

$$
\bar{\eta}=-\bar{\phi}_{t}, \quad B_{2}=\frac{1}{2} B^{2}+i B B_{t}, \quad \text { and } \quad B_{3}=\frac{3}{8} B^{3} .
$$

Detailed representations of temporal and spatial Schrödinger equations are given in 23]. The temporal evolution equations have nice properties to investigate temporal sequences of measurements obtained from large-area remote sensing systems. However, for a meaningful study of the spatial development of frequency dependent directional spread away from a wave maker in a basin it is desirable to employ spatial MNLS equation. In particular, when the measurements are in the form of simultaneously recorded time series of certain wave properties taken at different spatial locations, the spatial MNLS equation is more suitable than the temporal MNLS. Moreover, conventional analysis methods often use time series to estimate the basic directional parameters.

Provided that the steepness is small $\epsilon=k_{c} a_{c} \ll 1$, the bandwidth is narrow $|\Delta \mathbf{k}| / k_{c} \ll 1$, large depth $\left(k_{c} h\right)^{-1} \ll 1$, and neglecting terms of relative order of magnitude higher than $\epsilon^{4}$, we get the modified nonlinear Schrödinger (MNLS) equation. Here $a_{c}=\sqrt{2\langle\eta\rangle^{2}}$ is the characteristic amplitude, $|\Delta \mathbf{k}|$ is a characteristic modulation wave vector relative to the characteristic wave vector, 21] and $h$ is depth set to infinity for the present numerical simulations. The potential $\bar{\phi}$ of the induced mean flow is governed by the equations

$$
\begin{aligned}
\bar{\phi}_{z}=-\left(|B|^{2}\right)_{t} \quad \text { at } \quad z=0, \\
4 \bar{\phi}_{t t}+\bar{\phi}_{y y}+\bar{\phi}_{z z}=0 \text { for } \quad-\infty<z<0, \\
\bar{\phi}_{z}=0 \quad \text { at } \quad z \rightarrow-\infty .
\end{aligned}
$$


The first, second and third harmonic complex amplitudes of the potential, $A, A_{2}$ and $A_{3}$, will not be considered here. The slowly varying functions $\bar{\eta}$ and $\bar{\phi}$ contain the difference-frequency contributions. The modified nonlinear Schrödinger equation [41, 42] is the lowest order that can capture the behavior observed in [1]. The spatial evolution form of MNLS equation is given by [20, $21,24]$

$$
B_{x}+2 B_{t}+i B_{t t}-\frac{i}{2} B_{y y}+i|B|^{2} B-B_{t y y}-8|B|^{2} B_{t}-2 B^{2} B_{t}^{*}-4 i \bar{\phi}_{t} B=0,
$$

superscript $*$ is complex conjugate. We employ the numerical method of [43, 44] with periodic boundary conditions in time and transverse direction. Evolution in the $x$-direction is achieved with a splitting scheme in which the linear part of Eq. 24] is integrated exactly in Fourier space and the nonlinear part is integrated by finite differences. We employ temporal and transversal grids with $N_{t}=512$ and $N_{y}=256$ points for simulation of $B$. The computational domain is set to 200 peak periods in time and 100 peak wavelengths in transversal direction with discretization $\Delta \omega=1 / 200$ and $\Delta k_{y}=1 / 100$. However, a different grid size namely, $N_{t}=4096$ and $N_{y}=2048$ points, is employed for the reconstruction of the surface elevation using Eq. 21, in this case we set the discretization of the $(t, y)$-plane to be $\Delta t=\Delta y=0.3$. For the directional analysis, evolution results are extracted at ten positions for every $10 \lambda_{c}$ interval over a total length of about $100 \lambda_{c}$ in the $x$-direction. The computational efforts range from an hour to few hours on office computers.

All simulations are started by a JONSWAP frequency spectrum with $\gamma=3.3$, multiplied by directional distributions of the form [2]

$$
D(\theta)=D_{k}(k) \cos ^{k}\left(\theta-\theta_{1}\right), \quad \text { for } \quad\left|\theta-\theta_{1}\right| \leq \frac{\pi}{2},
$$

where $D_{k}(k)$ is a normalization factor. This distribution is slightly advantageous for MNLS-type simulations in comparison with cos- $2 s$, because it does not prescribe waves going backward. We recall that $\sigma_{1}=\sqrt{2 /(s+1)}$ for the cos- $2 s$-distribution, on the contrary there is no simple analytic expression for $k$ as a function of $\sigma_{1}$. Nevertheless, since the distribution in Eq. 25] and the 
cos- $2 s$-distribution are practically indistinguishable when $k=(s-1) / 2$ is larger than about 5 [2], one may use the approximation

$$
k=(s-1) / 2=\frac{1}{\sigma_{1}^{2}}-1,
$$

\subsection{Laboratory observations}

Full 3-D spectra were obtained from three-dimensional discrete Fourier transform in space and time of MARIN's $10 \times 10$-array data. To reduce spectral leakage, the Fourier transform has been tapered with a cosine-bell window. In addition, we have applied a three-dimensional moving average to smooth the 


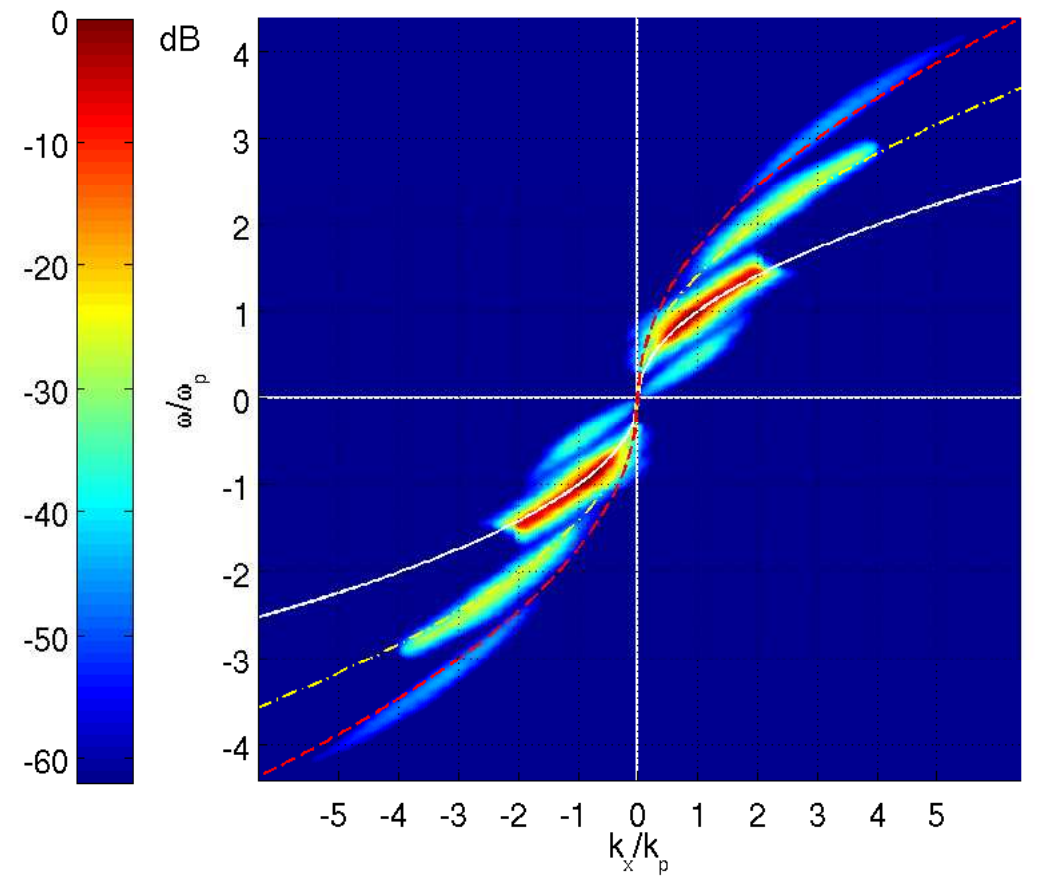

Figure 2: MNLS: $k_{x} \omega$-slice obtained from 3-D spectrum at $k_{y}=0$. Color scale in dB relative to the peak. White solid curve, deep water dispersion relation; yellow dash-dot curve, second order contribution; red dashed curve, third order contribution. 


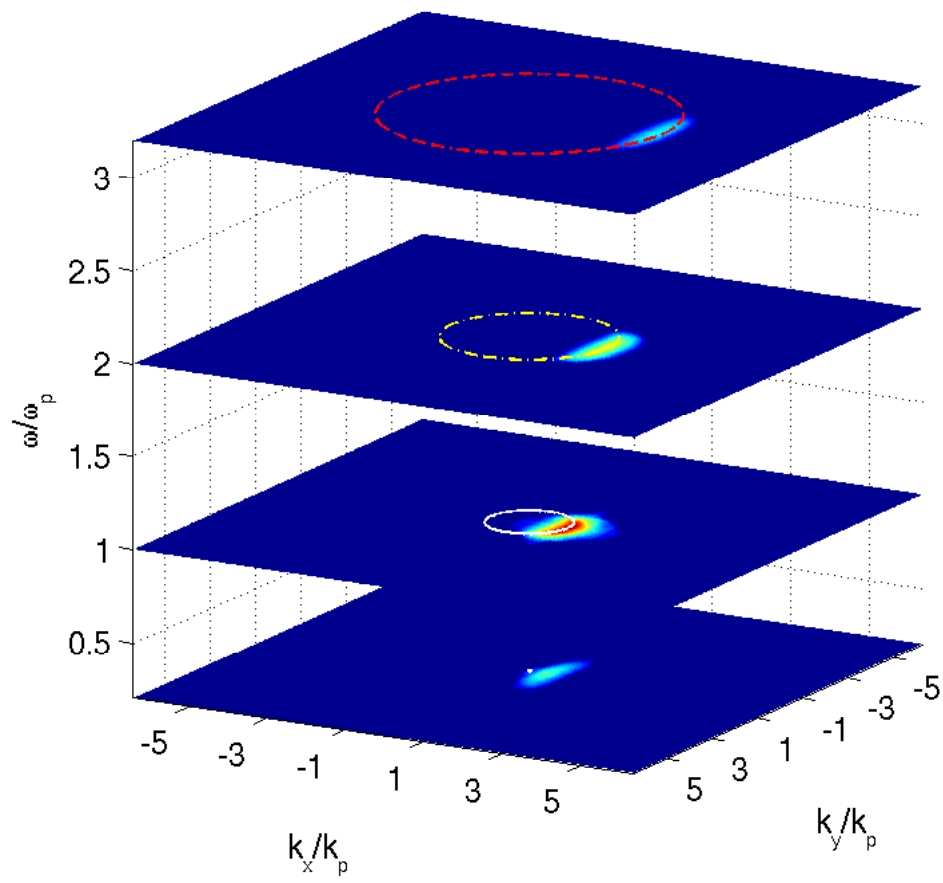

Figure 3: MNLS: Slices through $(\mathbf{k}, \omega)$-spectrum showing $\phi_{\omega}(\mathbf{k})$ for constant frequencies. White solid circle, deep water dispersion relation; yellow dash-dot circle, second order contribution; red dashed circle, third order contribution. 
resolution in the $\mathbf{k}$-plane is coarse, 40 neighboring slices have been averaged in the $\omega$-direction. The result is heavily smeared and biased compared to the corresponding distributions obtained from simulated data (see Fig. 3). Nevertheless, the maximum distribution lies outside the dispersion circle for $\omega<\omega_{p}$ and within the dispersion circle for $\omega>\omega_{p}$.
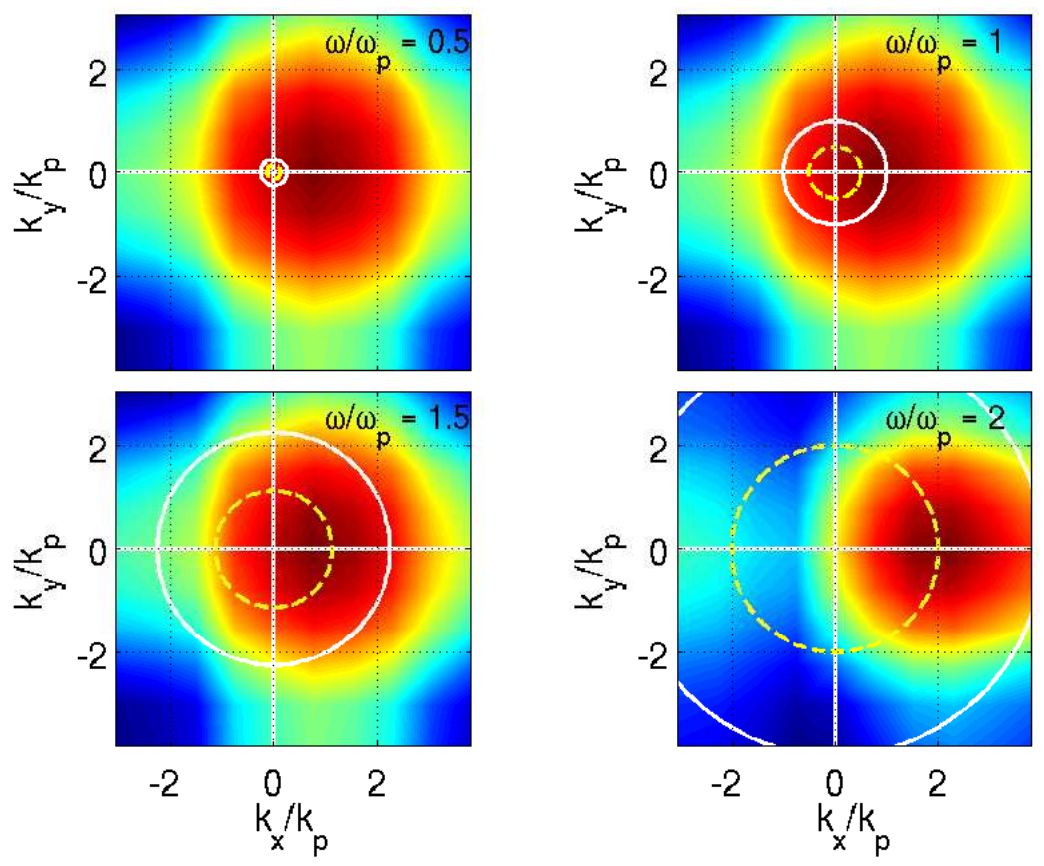

Figure 4: MARIN (location 2, full 3-D analysis, $\left.\sigma_{1 I}=19.1^{\circ}\right)$ : $\phi_{\omega}(\mathbf{k})$ for fixed $\omega$. All the plots are scaled with the maximum value of $\phi_{\omega}(\mathbf{k})$ for the given $\omega$. Solid circle, linear dispersion relation at specific frequency; dashed circle, second order contribution.

205

From the estimate of $\chi(\mathbf{k}, \omega)$, one may obtain the moments for each frequency and then determine the directional parameters as discussed in Sec. 2.1 The data may also be analyzed using the standard ML and IML algorithms. These provide estimates of the directional distributions, $D(\theta, \omega)$, from which we then determine the directional parameters. Alternatively, the time series data may be 
interpolated into HPR time series, and analyzed in that way, leading to estimates of the five basic moments. Figure 5 shows frequency spectra on the three sensors and estimated directional spectra, obtained by ML, IML and ME-B algorithms. The frequency spectra, in the upper left corner, are almost identical. Observe also the bend, slightly below twice the spectral peak, where the noise takes over the spectra. The spectra from the three algorithms are also very similar, with no distinct bi-modality which is perhaps masked by spatial aliasing in the high frequency region.
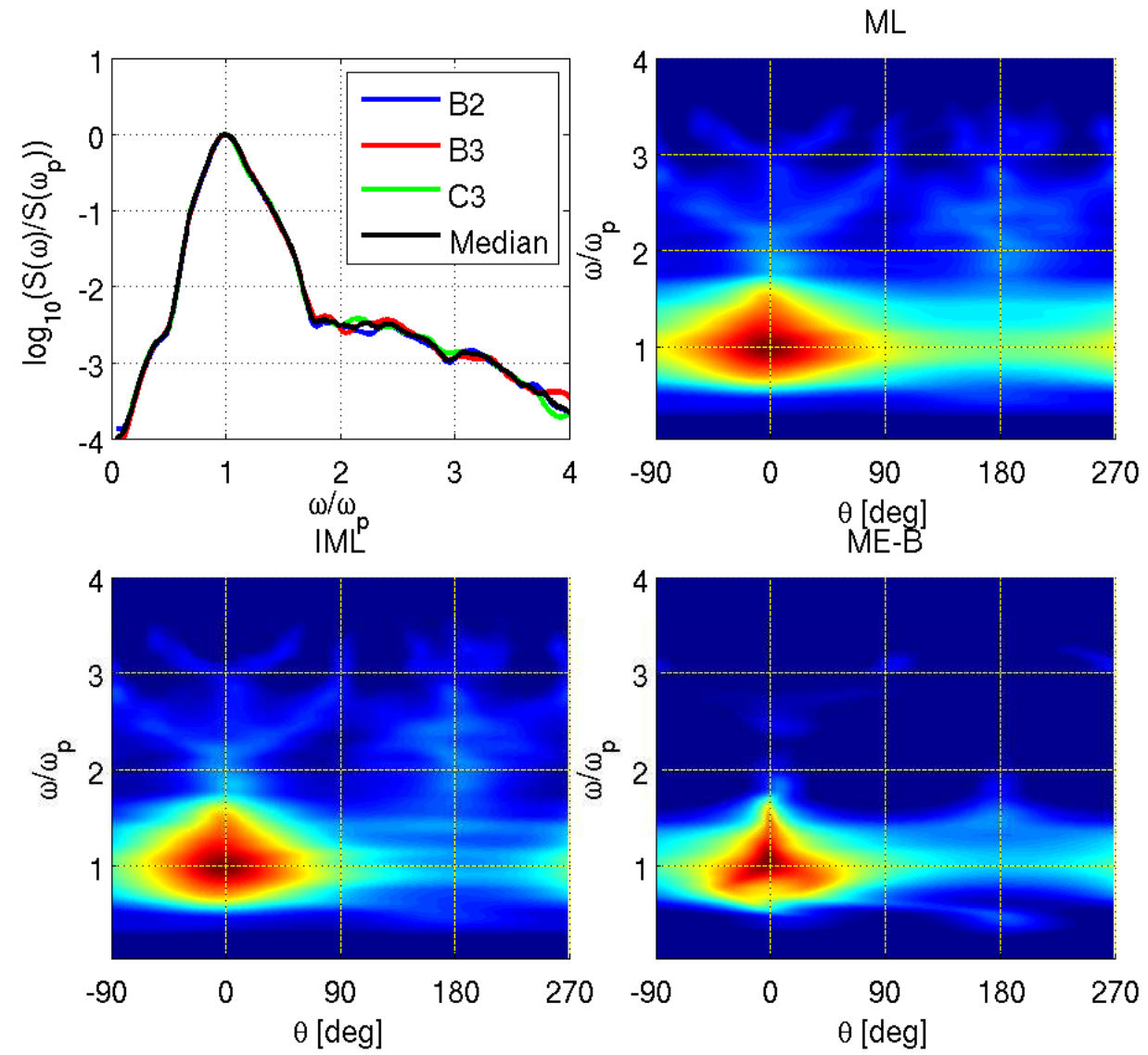

Figure 5: MARIN (location 2, array type $1, \sigma_{1 I}=19.1^{\circ}$ ): Frequency spectra on the three sensors and their median (upper left), ML, IML and ME-B directional spectra. 
In Fig. 6, estimates of the directional spread from the three wave conditions

HPR fit surprisingly well, in particular for the broader distributions.

Seven more datasets from each wave condition were analyzed to examine the spreading behavior more closely, in almost all of them, results were consistent and in agreement with the ones shown in Fig. 6 opment of the various wave parameters in the MARIN wave tank is shown in Fig. 7 for the broad spread $\left(\sigma_{1 I}=19.1^{\circ}\right)$. In each plot, the graphs represent results from six locations along the propagation direction designated by $1-4$, 


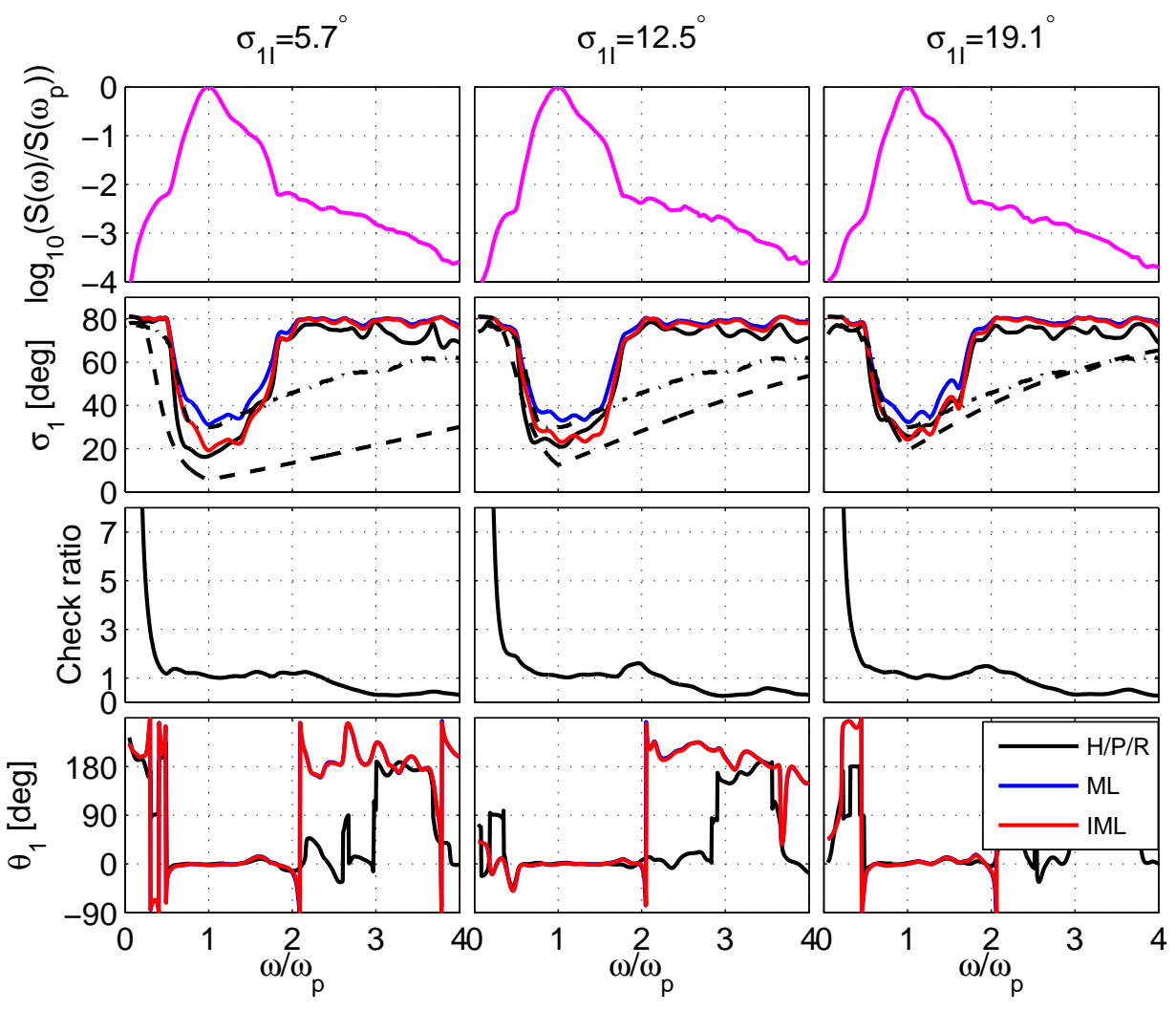

Figure 6: MARIN (location 2, array type 1): Frequency spectra (top row), directional spread (second row), check ratio (third row) and mean directions (bottom row) for narrow, medium and broad input spread. Black curve: standard triplet analysis (HPR); blue curve: ML analysis; red curve: IML analysis; dash-dot line: Ekofisk spread; dashed line: Mitsuyasu spread. The IML analysis is performed with $\omega_{R}=0.2$ and 3 iterations. 

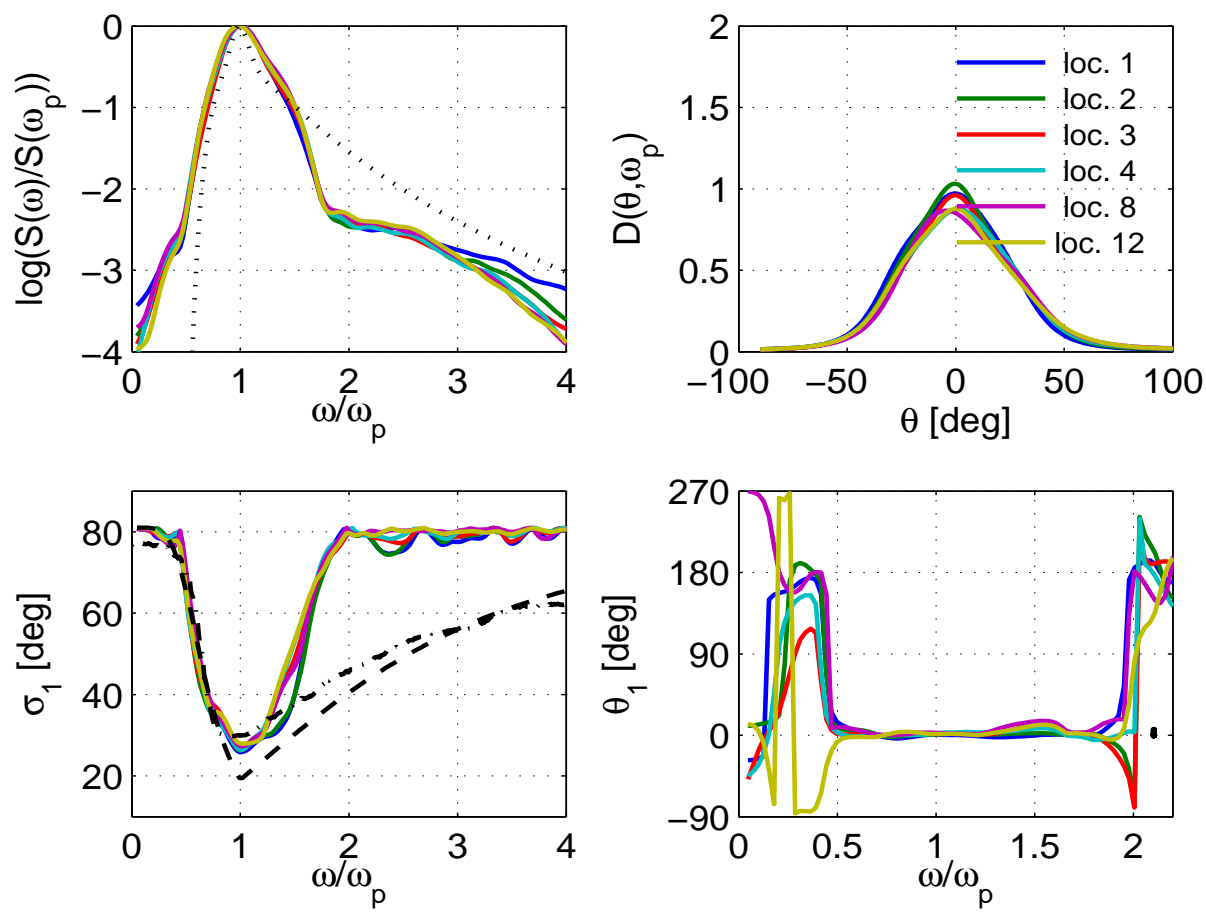

Figure 7: MARIN (ML on Array type 2, $\sigma_{1 I}=19.1^{\circ}$ ): Spatial evolution of frequency spectrum, directional distribution for peak frequency, directional spread, and the mean direction. Dotted line: input frequency spectrum; dash-dot line: Ekofisk spread; dashed line: Mitsuyasu spread.

8 and 12 in Fig. 1b, where the evolution distance between location 12 and 1 is about 14 peak wavelengths. Results are very similar, regardless of measurement locations, it is likely that the similarity observed is linked to a lack of spectral evolution. In the upper left corner, the input frequency spectrum is narrow compared to the estimate from the data. In the lower left corner, field directional spread from the Ekofisk laser array is shown. It is an averaged spread from ten records with large $H_{s}$. The MARIN spread deviates from the Ekofisk spread at about 1.5 the spectral peak and increases to maximum at twice the spectral peak due to an effect of spatial aliasing and apparent lack of spectral coherence, see Sec. 3.1 . 


\subsection{Field observations}

We shall consider estimates of directional spread and distributions based on the ML, IML and HPR methods, all described in Sec. 2.2. The ML and IML algorithms provide estimates of the directional distributions from which we then determine $\theta_{1}(\omega)$ and $\sigma_{1}(\omega)$, whereas the HPR method provides the four leading Fourier coefficients to, among others, the Burg-ME and ShannonME directional distributions. The Burg-ME produces two peaks when applied to the Fourier coefficients of, for instance, the cos- $2 s$ distribution. This peak splitting tendency is sometimes considered to be a weakness of the method [2]. We leave the details to a separate paper and concentrate here on illustrating some of the results in Fig. 8 and 9] Frequency spectra of the three lasers (L) are almost identical and the agreement between the mean direction is perfect for the range of frequencies up to about four times the spectral peak. Above about four times the spectral peak, the ML and IML directions veer off from the HPR direction. This is a well known effect of spatial aliasing in the ML algorithms.

275 Estimates of the directional spread are shown on the upper left corner, there is a significant bias in the directional spread between ML and HPR estimates, whereas the IML and HPR spreads are surprisingly similar up to about four times the spectral peak. The HPR method gives the opportunity to study the estimator for the spread without making any assumptions about LWT. Besides,

it can be used as a benchmark to evaluate other directional analysis methods when possible.

For the directional spectra, the estimate by the standard ML differs significantly from both IML and ME estimates. The IML and ME spectra show more details compared to the rather smeared ML spectrum. Many ME analyzed data suggest a bimodal directional distribution for high frequencies, and in many situations, this bi-modality is even observed in the IML spectra for moderately high frequencies and in the Shannon based ME results for relatively higher frequencies. The highly different treatment for the data analysis of ME and IML suggests that in these situations, the bi-modality is real. However, in many circumstances, the peaks in the Burg ME distributions are pronounced 

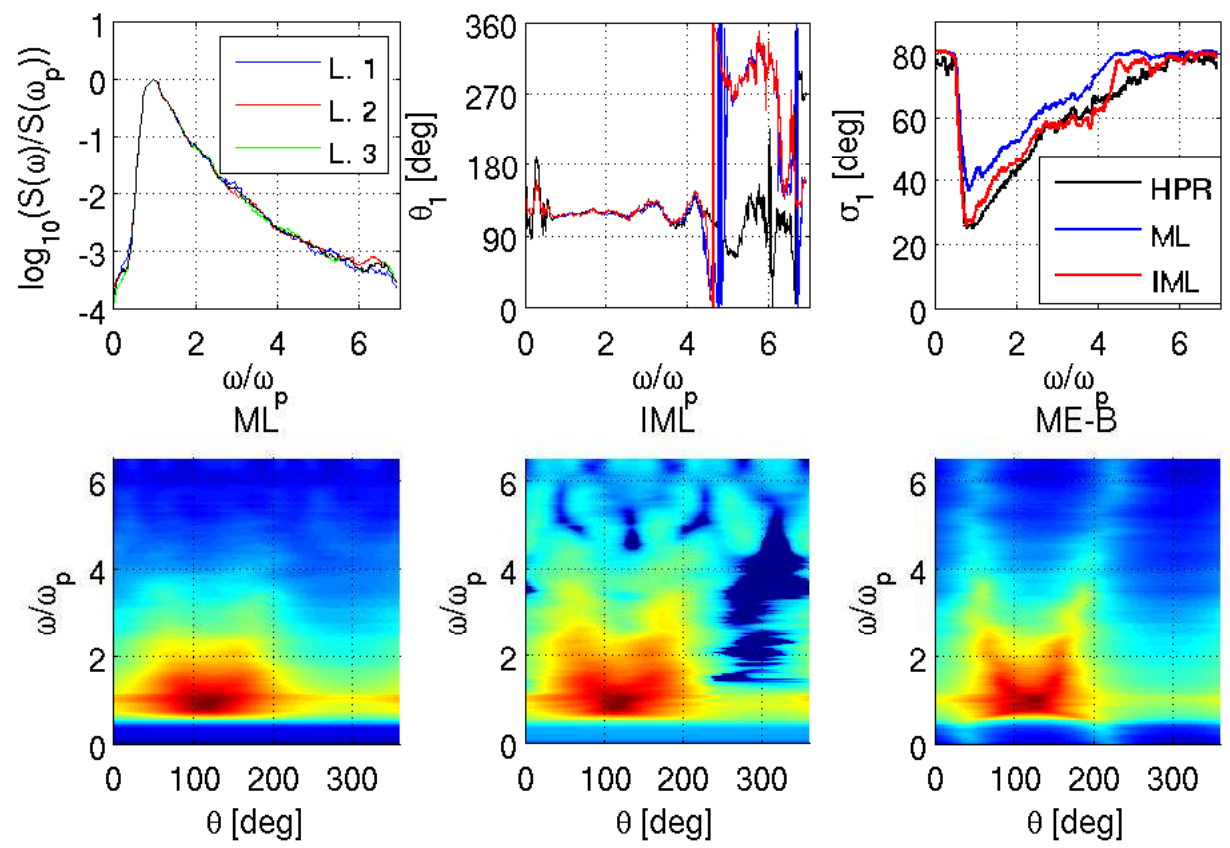

Figure 8: Ekofisk data recorded on the $12^{\text {th }}$ of Oct. 2004 at 12:00, $H_{s}=3.58 \mathrm{~m}$. Upper left: Frequency spectra for the three input time series. Upper centre: Mean wave directions. Upper right: Directional spread. Lower row: Directional spectra. 
compared to the moderate peaks observed in both IML and the Shannon based ME distributions ( see Fig. 9).

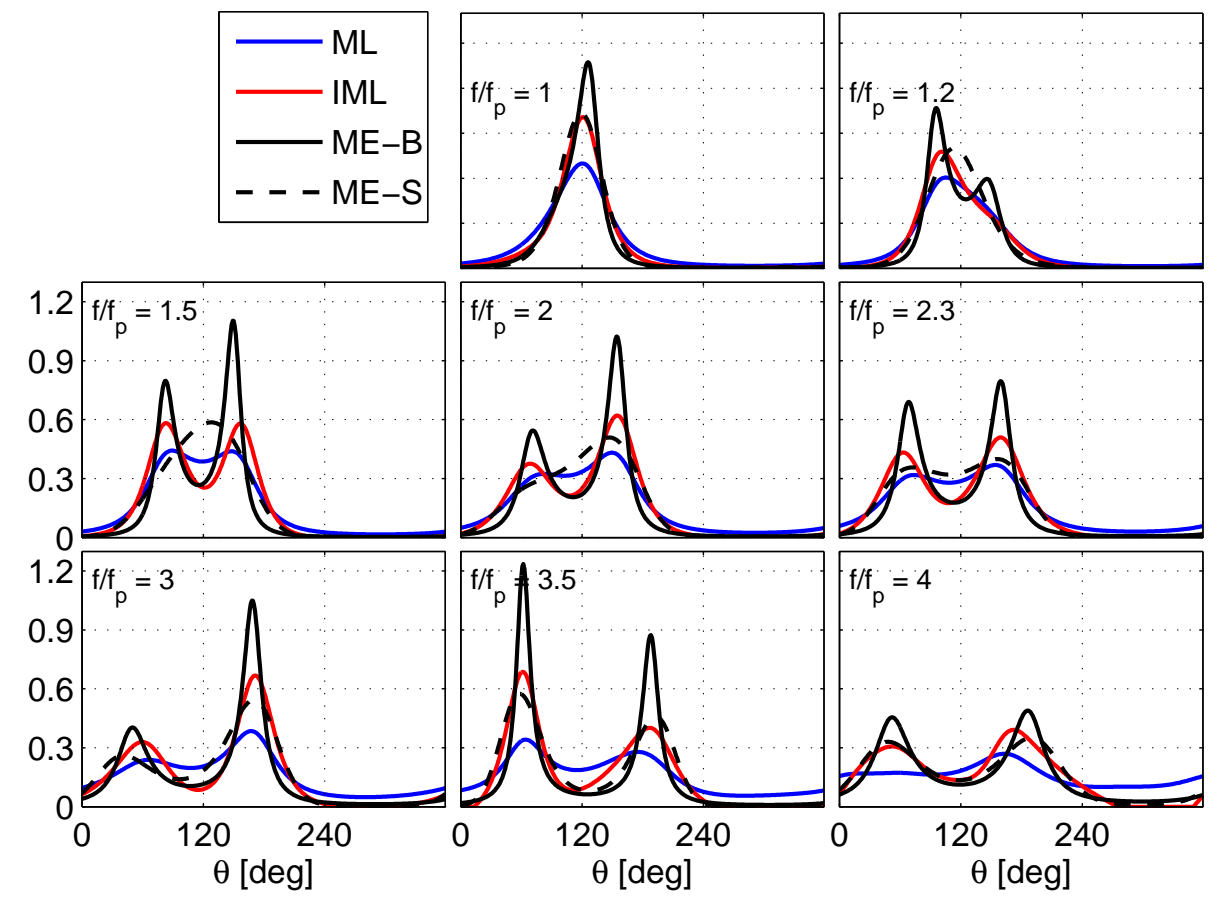

Figure 9: Ekofisk data recorded on the $12^{\text {th }}$ of Oct. 2004 at $12: 00, H_{s}=3.58 \mathrm{~m}$. Directional distributions, $D(\theta, \omega)$, for selected frequencies.

\subsection{Linear simulation with nonlinear reconstruction}

In order to show that the reconstruction in Eq. 21, together with linear wave evolution, recovers the frequency dependent spread reported in [1], we present in Fig. 10] an HPR analysis of results from simulations carried out for infinitely deep water and with steepness $\epsilon=0.05$ and spread $\sigma_{1 I}=5.7^{\circ}, 12.5^{\circ}$ and $19.1^{\circ}$ similar to the MARIN experiments. In the simulations, only the linear part of Eq. 24 is integrated to discard the dynamic nonlinearity; on the other hand, the reconstruction in Eq. 21 takes the static nonlinear contributions into account. The estimated directional spread is different in shape from the input to the 
model, it is strongly dependent on frequency and remains the same everywhere in the numerical basin.

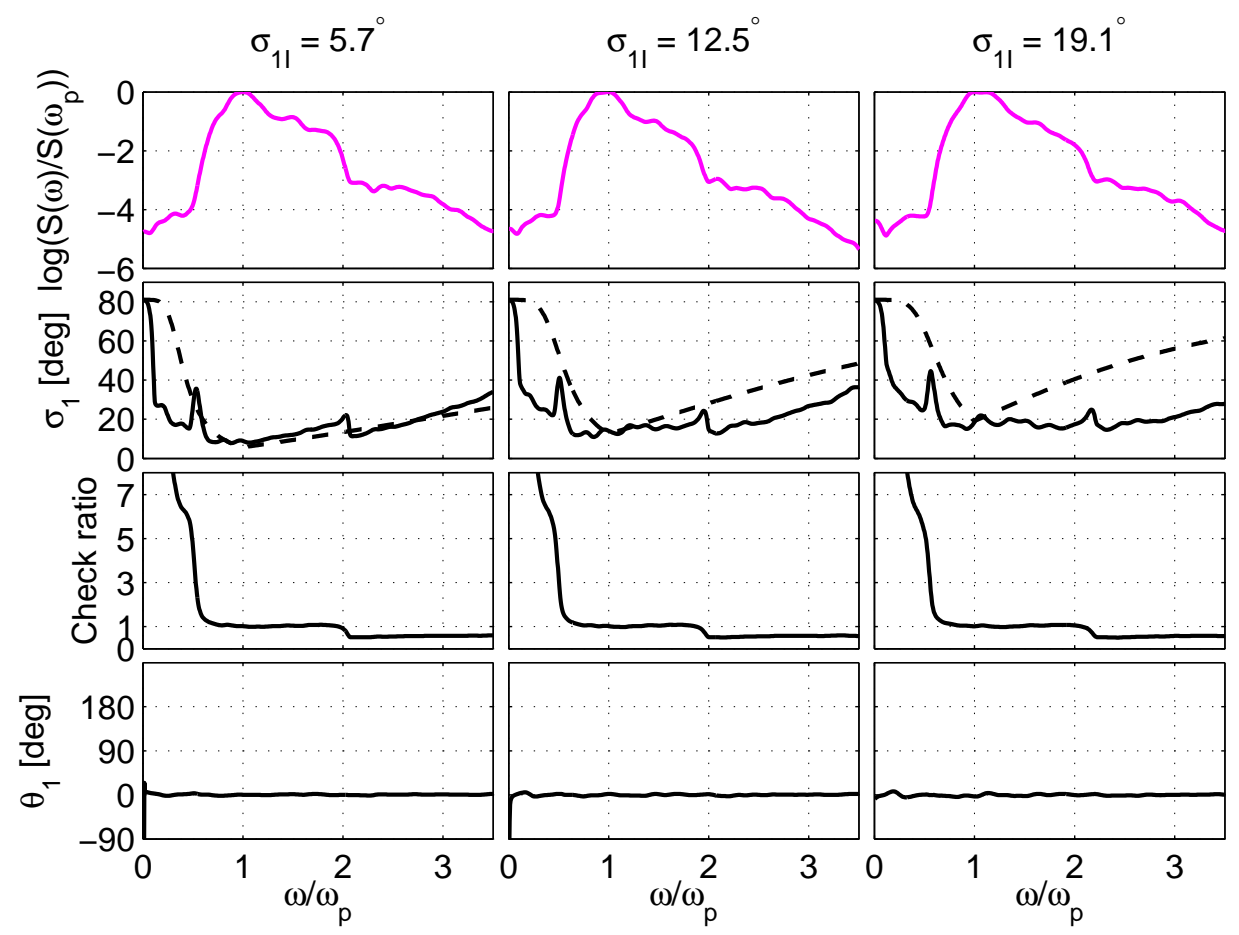

Figure 10: Simulation with the linear part of Eq. 24 together with reconstruction according to Eq. 21 Similar to Fig. 6 with the HPR method alone. Dashed line: Mitsuyasu spread.

For the narrow distribution $\left(\sigma_{1 I}=5.7^{\circ}\right)$, the spread clearly increases towards both high and low frequencies, whereas, for broad distribution $\left(\sigma_{1 I}=19.1^{\circ}\right)$ the increase toward the high frequencies is quite weak. In all of the above cases, the minimum spread lies below the spectral peak. The check ratio, in the middle row, shows a strong increase towards the low frequencies and a drop from 1 for frequencies higher than twice the spectral peak, where the second order spectral contribution is expected to dominate.

The numerical experiment has been checked by not including $\bar{\eta}$ in the reconstruction equation, Eq. 21. The resulting spread and check ratio did not show frequency dependent variations demonstrating that it is primarily the contribu- 
tion from $\bar{\eta}$ that produces the directional spread reported in [1]. Except for very

wide spectrum the agreement between our results and results in [1] is quite good. The observed behavior in the spread and check ratio, below the spectral peak, is therefore originated from second order difference-frequency contributions.

\subsection{Nonlinear simulation}

Finally we show numerical simulations using the full eqs. (21)-(24) demonstrating that the establishment of frequency dependent directional spread is not only a matter of static nonlinear reconstruction. In Fig. 11 the input directional spread has been set to $5.7^{\circ}, 12.5^{\circ}$ and $19.1^{\circ}$, and the steepness has been set to 0.05 and 0.1 . With the dynamic nonlinearity in the evolution equation, the directional spread is found to grow with an increase in input wave steepness, the minimum spread is also moved toward the spectral peak. This is shown even more clearly in Fig. 12 which employs six different steepnesses between 0.05 to 0.1 , inclusive.

Depending on the steepness of the waves, the numerical simulations demonstrate the necessary propagation distance required for sufficient development of the spread. As seen in Fig. 11 for $\epsilon=0.05$ the directional spread, $\sigma_{1}(\omega)$, does not change significantly with evolution distance. This result is supported by the observations from the MARIN experiment. For $\epsilon=0.1$, the directional spread develops over a distance of 80 peak wavelengths before it becomes nearly stationary. We thus draw the conclusion that the distance for the frequency dependent directional spread to become fully established increases as the steepness increases.

From Fig. 11 it can be appreciated that the crest length decreases with increasing propagation distance. From Fig. 12 it can furthermore be appreciated that this effect is enhanced with increasing input steepness.

\section{Conclusion}

We have found both experimental and numerical evidence that a frequency dependent directional spread naturally develops from an initial state of no fre- 


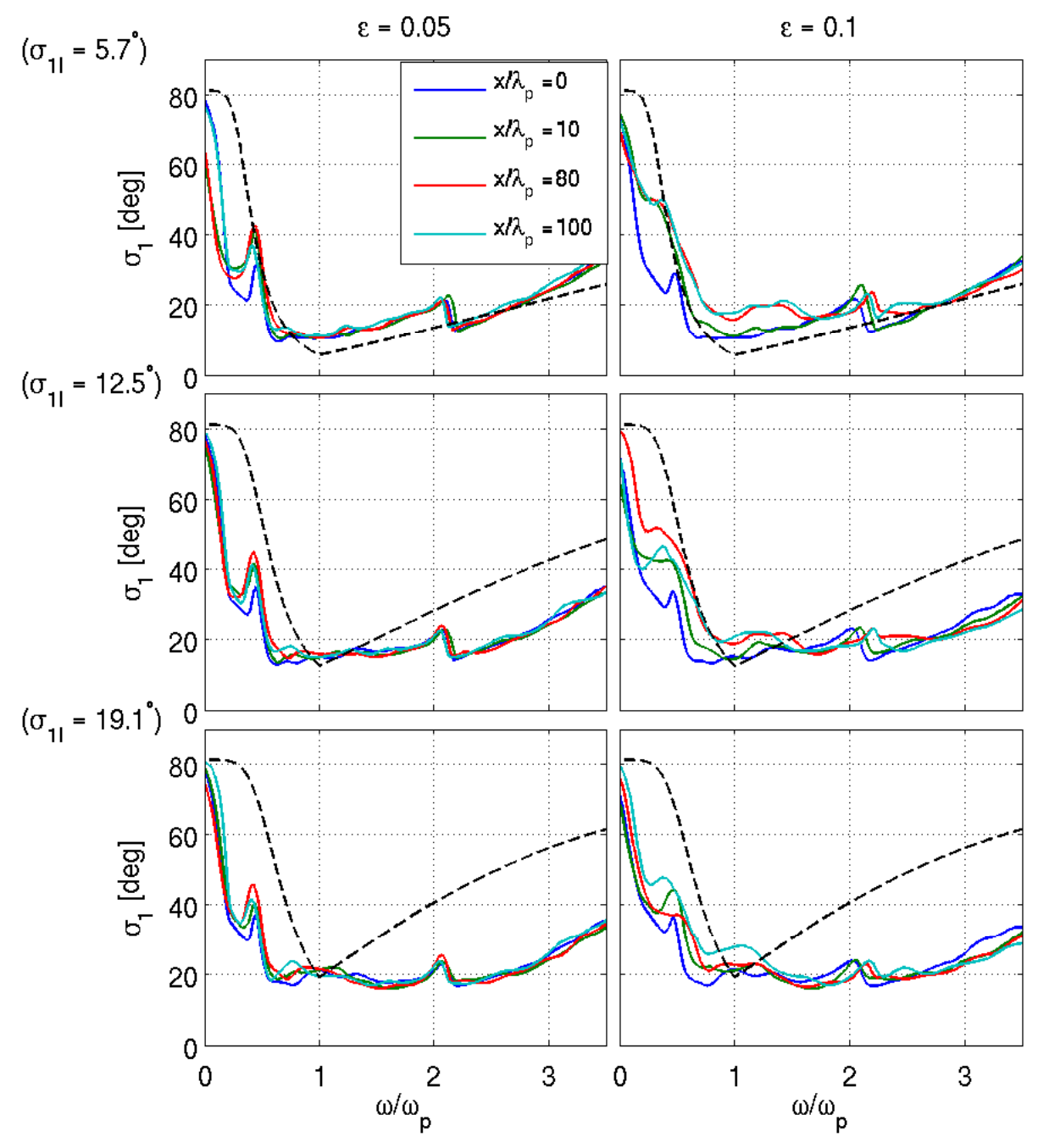

Figure 11: MNLS: Effects of dynamic nonlinearity and wave steepness on the development of the directional spread for $\sigma_{1 I}=5.7^{\circ}, 12.5^{\circ}$ and $19.1^{\circ}$; dashed line, Mitsuyasu spread. 


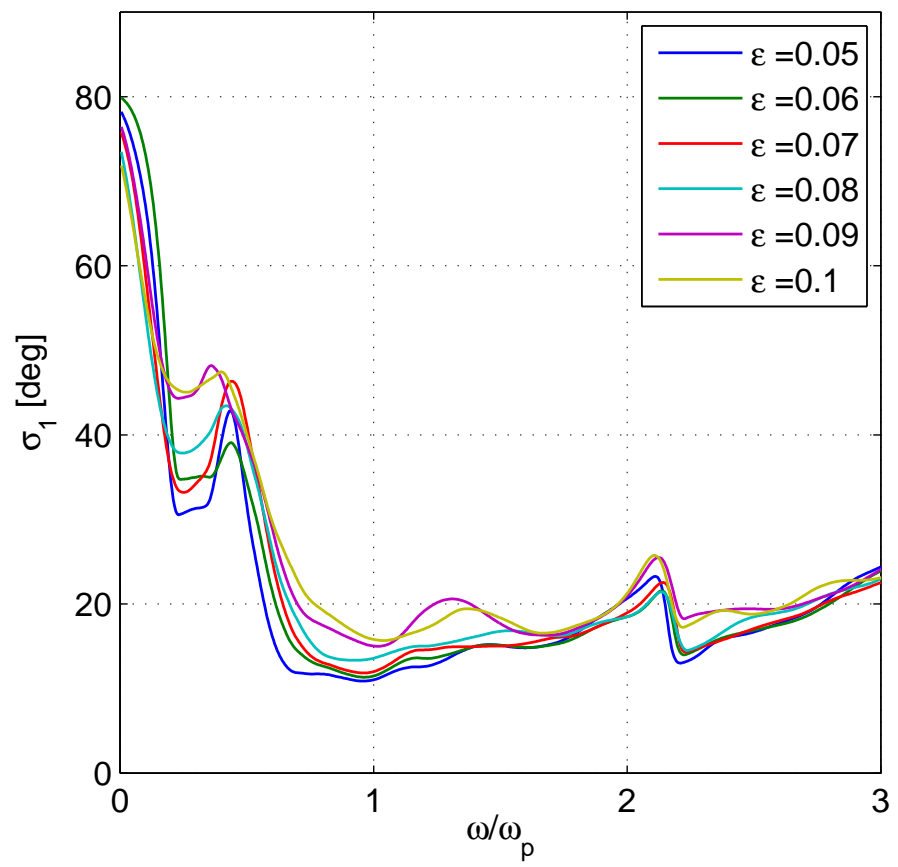

Figure 12: MNLS: Directional spread at 100 peak wavelengths from the numerical wave paddle. Input spread $\sigma_{1 I}=5.7^{\circ}$ and steepness $\epsilon=0.05,0.06,0.07,0.08,0.09$ and 0.1 . 
quency dependence. The frequency dependence is partly a consequence of static nonlinear contributions which act instantaneously, and partly a consequence of dynamic nonlinear contributions which need some evolution distance to develop. The static contribution occurs primarily below spectral peak, the dynamic contribution occurs both above and below spectral peak. The necessary distance for developing a steady frequency dependent directional spread seems to increase with increasing steepness of the wave field.

An important consequence of these observations is that it is not necessary to generate a frequency dependent directional spread in wave basins since the wave field will tend to develop an adequate frequency dependent directional spread by itself, care just needs to be taken that the wave field has been allowed to develop over a sufficiently long distance that a steady frequency dependence has 355 been established.

The analysis of datasets from the Ekofisk field indicates that a large fraction of the directional wave spectra have a bimodal character above the spectral peak. The bi-modality is observed for all four algorithms employed in the paper suggesting that the bi-modality is not limited to, as often claimed, an artifact of the Burg ME algorithm.

Acknowledgments. For the present study use has been made of the extensive 3D wave measurements that have been conducted by MARIN in Wageningen as part of the On Board Wave and Motion Estimator (OWME) JIP. The authors would like to thank MARIN and the participants in the OWME JIP (Gusto, Kongsberg, SBM Seaflex, Sirehna, Statoil and Total) for making the data available for this study. The field data are from the laser array at the Ekofisk field in the North Sea and used by permission of ConocoPhillips Inc.

This research has been funded by the Research Council of Norway, RCN, and the University of Oslo through projects RCN 225933 and RCN 214556. 
[1] H. E. Krogstad, (2002) Second order wave spectra and heave/slope wave measurements, in: Proc. Ocean wave measurement and analysis, San Francisco, Ed. B. L. Edge and J. M Hemsley, 2001, pp. 288-296.

[2] K. Kahma, D. Hahuser, H. E. Krogstad, S. Lehner, J. A. J. Monbaliu, L. R. Wyatt, Measuring and analysing the directional spectra of ocean waves, Vol. EUR 21367, ISBN 92-898-0003-8, 2005.

[3] A. Benetazzo, Measurements of short water waves using stereo matched image sequences, Coastal Engineering 53 (2006) 1013-1032.

[4] A. Benetazzo, F. Fedele, G. Gallego, P. C. Shih, A. Yezzi, Offshore stereo measurements of gravity waves, Coastal Engineering 64 (2012) 127-138.

[5] C. J. Zappa, M. L. Banner, H. Schultz, J. R. Gemmrich, R. P. Morison, D. A. LeBel, T. Dickey, An overview of sea state conditions and air-sea fluxes during radyo, Journal of Geophysical Research: Oceans 117 (2012) C00H19.

[6] P. Sutherland, W. K. Melville, Field measurements and scaling of ocean surface wave-breaking statistics, Geophysical Research Letters 40 (2013) 3074-3079.

[7] J. C. Nieto-Borge, S. G. R., K. Hessner, K. Reichert, S. C. Guedes, Estimation of sea state directional spectra by using marine radar imaging of sea surface, Proceedings of the ETCE/OMAE2000 joint conference, New Orleans, LA (2000) OMAE2000/S\&R-6120.

[8] M. S. Longuet-Higgins, S. Michael, D. E. Cartwright, N. D. Smith, Observations of the directional spectrum of sea waves using the motions of a floating buoy, in: Ocean Wave Spectra, proceedings of a conference, Easton, Maryland, National Academy of Sciences, Prentice-Hall, 1963, pp. $111-136$. 
[9] K. C. Ewans, Observations of the Directional Spectrum of Fetch-Limited Waves, J. Phys. Oceanogr. 28 (1998) 495-512.

[10] H. E. Krogstad, S. F. Barstow, O. Haug, D. J. H. Peters, Directional distributions in wave spectra, Proc. Waves'97 Ocean wave measurements and analysis 2 (1998) 883-895.

[11] H. E. Krogstad, S. F. Barstow, Directional distributions in ocean wave spectra, in: Proc. ninth International offshore and polar engineering conference (Brest, France, May 30-June 4 1999), Vol. III, 1999.

[12] J. A. Ewing, A. K. Lang, Directional spectra of seas near full development, Journal of Physical Oceanography 17 (1987) 1696-1706.

[13] D. E. Hasselmann, M. Dunckel, J. E. Edwing, Directional wave spectra ovserved during JONSWAP 1973, J. Phys. Oceanogr. 10 (1980) 1264-1280.

[14] H. Mitsuyasu, F. Tasai, T. Suhara, S. Mizuno, M. Onkusu, T. Honda, T. Rukiiski, Observations of the directional spectrum of ocean waves using a cloverleaf buoy, J. Phys. Oceanogr. 5 (1975) 751-761.

[15] W. H. H. M. A. Donelan, J. Hamilton, Directional spectra of windgenerated waves, Philosophical Transactions of the Royal Society of London. Series A, Mathematical and Physical Sciences 315 (1534) (1985) 509562.

[16] G. Z. Forristall, K. C. Ewans, Worldwide measurements of directional wave spreading, Journal of Atmospheric and Oceanic Technology 15 (2) (1998) 440-469.

[17] A. K. Laing, Nonlinear properties of random gravity waves in water of finite depth, Journal of physical oceanography 16 (1986) 2013-2030.

[18] K. B. Dysthe, K. Trulsen, H. E. Krogstad, H. Socquet-Juglard, Evolution of a narrow-band spectrum of random surface gravity waves, Journal of Fluid Mechanics 478 (2003) 1-10. 
[19] C. E. Schmittner, J. Scharnke, W. Pauw, J. van den Berg, J. Hennig, New methods and insights in advanced and realistic basin wave modelling, in: ASME 32nd International Conference on Ocean, Offshore and Arctic Engineering, American Society of Mechanical Engineers, 2013.

[20] K. Trulsen, K. B. Dysthe, Frequency downshift in three dimensional wave trains in a deep basin, Journal of Fluid Mechanics 352 (1997) 359-373.

[21] K. Trulsen, Spatial evolution of water surface waves, Proc. of Waves, Paper number 127 (2005) 1-10.

[22] K. Trulsen, Weakly nonlinear and stochastic properties of ocean wave fields. application to an extreme wave event, in: J. Grue, K. Trulsen (Eds.), Waves in Geophysical Fluids, Vol. 489 of CISM International Centre for Mechanical Sciences, Springer Vienna, 2006, pp. 49-106.

[23] K. Trulsen, Weakly nonlinear sea surface waves freak waves and deterministic forecasting, in: G. Hasle, K. A. Lie, E. Quak (Eds.), Geometric Modelling, Numerical Simulation, and Optimization, Springer Berlin Heidelberg, 2007, pp. 191-209.

[24] A. Toffoli, O. Gramstad, K. Trulsen, J. Monbaliu, E. Bitner-Gregersen, M. Onorato, Evolution of weakly nonlinear random directional waves: laboratory experiments and numerical simulations, Journal of Fluid Mechanics 664 (2010) 313-336.

[25] I. R. Young, L. A. Verhagen, M. L. Banner, A note on the bimodal directional spreading of fetch-limited wind waves, Journal of Geophysical Research: Oceans 100 (1995) 773-778.

[26] D. W. Wang, P. A. Hwang, Evolution of the bimodal directional distribution of ocean waves, Journal of Physical Oceanography 31 (2001) 12001221. 
450

[27] A. Toffoli, M. Onorato, E. M. Bitner-Gregersen, J. Monbaliu, Development of a bimodal structure in ocean wave spectra, Journal of Geophysical Research: Oceans 115 (2010) C03006.

[28] H. E. Krogstad, K. Trulsen, Interpretations and observations of ocean wave spectra, Ocean Dynamics 60 (2010) 973-991.

[29] K. V. Mardia, Statistics of Directional Data, Probability and Mathematical Statistics a Series of Monographs and Textbooks, Academic Press, 1972.

[30] H. E. Krogstad, Maximum likelihood estimation of ocean wave spectra from general arrays of wave gauges, Modelling, Identification and Control 9 (1988) 81-97.

[31] Y. Goda, Random Seas and Design of Maritime Structures, Advanced series on ocean engineering, World Scientific, 2010.

[32] H. E. Krogstad, R. L. Gordon, M. C. Miller, High-resolution directional wave spectra from horizontally mounted acoustic doppler current meters, J. Atmos. Oceanic Technol. 5 (1988) 340-352.

[33] S. S. Pawka, Island shadows in wave directional spectra, Journal of Geophysical Research: Oceans 88 (1983) 2579-2591.

[34] H. E. Krogstad, Maximum likelihood estimation of ocean wave spectra from general arrays of wave gauges, Modelling, Identification and Control 9 (1988) 81-97.

[35] M. K. Ochi, Ocean Waves: The Stochastic Approach (Cambridge Ocean Technology Series), Cambridge University Press, 2005.

[36] A. Lygre, H. E. Krogstad, Maximum entropy estimation of the directional distribution in ocean wave spectra, Journal of Physical Oceanography 16 (1986) 2052-2060. 
[44] E. Y. Lo, C. C. Mei, Slow evolution of nonlinear deep water waves in two horizontal directions: A numerical study, Wave Motion 9 (1987) 245-259. 